\title{
Remission of late-onset post-heart transplantation lymphoproliferative disorder following treatment with rituximab and modified mini-CHOP chemotherapy: A case report
}

\author{
QIANG HUANG, TIANXIN YANG, XING JIN, XUMING NI, HAIYAN QI and ZHIKUN YAN \\ Department of Hematology and Cardiothoracic Surgery, Zhejiang Provincial People's Hospital, \\ Hangzhou, Zhejiang 310014, P.R. China
}

Received February 7, 2015; Accepted April 5, 2016

DOI: $10.3892 / e t m .2016 .3310$

\begin{abstract}
Post-transplant lymphoproliferative disorder (PTLD) is one of the most frequent secondary malignancies that can follow immunosuppressive therapy for solid organ transplantation, and may result in severe morbidities and even mortality. A middle-aged Han Chinese patient, prescribed with immunosuppressive cyclosporine and prednisone, developed PTLD that manifested as a painless cervical lymph node enlargement, 12 years following heart transplantation. Histology revealed monomorphic B-cell PTLD (diffuse large-cell lymphoma); as a result the immunosuppressive regimen of the patient was changed to tacrolimus and mycophenolate mofetil. In addition, the patient was changed to 6-cycle rituximab with a modified mini-CHOP (R-mini-CHOP) regimen for induction, and 8-cycle quarterly rituximab treatment and maintenance therapy. R-mini-CHOP therapy was well tolerated, and no allograft rejection occurred. The patient exhibited clinical remission as demonstrated by the results of the positron emission tomography-computed tomography at the 5-year follow-up visit following R-mini-CHOP therapy. In conclusion, R-mini-CHOP therapy following reduced immunosuppression is effective and safe for the treatment of late-onset PTLD following heart transplantation.
\end{abstract}

\section{Introduction}

Post-transplant lymphoproliferative disorder (PTLD) is a well-recognized and potentially life-threatening morbidity that can occur following transplantation of allogeneic hematopoietic stem cells and solid organs, including the heart (1-3). It

Correspondence to: Dr Qiang Huang, Department of Hematology and Cardiothoracic Surgery, Zhejiang Provincial People's Hospital, 158 Shangtang Road, Hangzhou, Zhejiang 310014, P.R. China E-mail: zjsrmyy@zjwst.gov.cn

Key words: lymphoproliferative disorder, heart transplantation, tomography-computed tomography, diffuse large-cell lymphoma is estimated to occur in 1-6.3\% cardiac recipients, and manifests as a spectrum of lymphoproliferative disorders, from benign lymphoid hyperplasia to invasive lymphoma (1-3). An Epstein-Barr virus (EBV) infection resulting from post-transplantation use of immunosuppressive agents is understood to drive the neoplastic pathogenesis of B cells in PTLD (2). Treatment for PTLD primarily includes the reduction or cessation of immunosuppressive therapy, anti-EBV therapy, anti-neoplastic therapy, such as chemoradiation therapy and surgery, and immunotherapy (4).

Two subtypes of PTLD, early- and late-onset PTLD, occur within the first year post-transplantation and afterwards, respectively, and exhibit distinct pathological profiles and prognoses $(5,6)$. It has been reported that EBV-positive patients are more frequently afflicted with early-onset PTLD than their EBV-negative counterparts $(5,6)$. Conversely, late-onset PTLD occurs more frequently in older patients who are typically EBV-negative, following a longer latency period; these patients generally have a poor treatment response and unfavorable prognosis, as late-onset PTLD frequently acts like aggressive lymphoma (7).

The present study reports a case of an EBV-negative, middle-aged Han Chinese man who developed late-onset PTLD 12 years after heart transplantation. Previous treatment with cyclosporine and prednisone was replaced by tacrolimus and mycophenolate mofetil. Rituximab (a monoclonal CD20 antibody) and a modified mini-CHOP (R-mini-CHOP) chemotherapy regimen was well tolerated and resulted in a 5-year clinical remission, free of allograft rejection.

\section{Case report}

A 54-year-old Han Chinese patient received orthotopic heart transplantation due to a diagnosis of end-stage dilated cardiomyopathy at the Zhejiang Provincial People's Hospital (Hangzhou, China) in June 1997. The patient underwent an uneventful perioperative course and remained on immunosuppressive therapy with cyclosporine and prednisone. The patient exhibited a good general condition free of allograft rejection, infection, or other clinically significant transplant-associated complications. However, at a follow-up visit in April 2009, the patient complained of multiple, bilateral, painless, cervical 
masses, in the absence of other discomforts such as fever, toothache, cough or dyspnea. These masses were 1-3 cm in size and less mobile on palpation, and the local skin showed no redness or rash. Histologic examination with hematoxylin (Regal Biotechnology Co., Ltd., Bengbu, China) and eosin (Shanghai Xinsheng Chemical Technology Co., Ltd., Shanghai, China) was performed on a paraffin-embedded lymph node biopsy specimen ( $4 \mu \mathrm{m}$; Leica RM2135 microtome; Leica Microsystems GmbH, Wetzlar, Germany), and showed non-Hodgkin lymphoma-diffuse large B-cell lymphoma (DLBCL; Fig. 1A) under the microscope (BX5; Olympus Corporation, Tokyo, Japan). Immunohistochemistry was performed on lymph node paraffin-embedded tissue (4 $\mu \mathrm{m})$ by blocking with hydrogen peroxide, followed by incubation with primary antibodies against CD20 (1:150; rabbit monoclonal/polyclonal; cat. no. EP7), CD10 (1:50; rabbit monoclonal; cat. no. SP67), CD15 (1:200; mouse monoclonal; cat. no. MMA+BY87), CD3 (1:100; rabbit monoclonal; cat. no. EP41), CD30 (1:150; rabbit monoclonal; cat. no. EP154), CD38 (1:150; mouse monoclonal; cat. no. SPC32), CD79a (1:150; rabbit monoclonal; cat. no. EP82), CD45RO (1:150; mouse monoclonal; cat. no. UCHL1), PAX-5 (1:50; rabbit monoclonal; cat. no. EP156), BCL-6 (1:100; mouse monoclonal; cat. no. LN22), MUM-1 (1:200; rabbit monoclonal; cat. no. EP190), ALK (1:150; mouse monoclonal; cat. no. OT1/A4), TDT (1:150; mouse monoclonal; cat. no. SEN28), Ki67 (1:100; rabbit monoclonal; cat. no. EP5), kappa (1:150; mouse monoclonal; cat. no. CH15), lambda (1:150; mouse monoclonal; cat. no. SHL53) and EMA (1:150; mouse monoclonal; cat. no. GP1.4) for $15 \mathrm{~h}$ at $4^{\circ} \mathrm{C}$ (all purchased from OriGene Technologies, Inc. (Rockville, MD, USA). The results identified strong immuno-positivity for CD20 and CD79a (Table I) using ABC series (avidin-biotin complex; Vector Laboratories, Ltd., Peterborough, UK) and a microscope (BX5; Olympus Corporation). Bone marrow examination showed mild marrow hyperplasia (Fig. 1B), and chest/abdominal computed tomography (GE HiSpeec TC/i; The General Electric Company, Coventry, UK) detected no distant metastasis. Thus, a diagnosis of monomorphic PTLD-DLBCL was established based on the previous history of heart transplantation and medication with immunosuppressive agents, in accordance with the World Health Organization classification of PTLD (8). The present study was approved by the Ethics Committee of Zhejiang Provincial People's Hospital

Routine hematologic (Sysmex 2100; Sysmex Corporation Co., Ltd., Kobe, Japan), clinical biochemistry (AU5800; Beckman Coulter, Brea, CA, USA), serologic (Abbott i2000; Beckman Coulter), and virologic (EBV and cytomegalovirus) tests (Euroimmun UK, Ltd., London, UK) showed no clinically significant abnormality. The immunosuppressive regimen of the patient was changed to dose-reduced tacrolimus ( $2 \mathrm{mg}$ daily) and mycophenolate mofetil (1.5 g daily) prior to the initiation of chemotherapy. A rituximab (Roche Diagnostics, Basel, Switzerland) and R-mini-CHOP chemotherapy regimen for non-Hodgkin lymphoma was administered as follows: $375 \mathrm{mg} / \mathrm{m}^{2}$ rituximab, $400 \mathrm{mg} / \mathrm{m}^{2}$ cyclophosphamide (Jiangsu Hengrui Medicine Co., Ltd., Shanghai, China), $30 \mathrm{mg} / \mathrm{m}^{2}$ pirarubicin (Shenzhen Wanle Pharmaceutical Co., Ltd., Shenzen, China), and $1 \mathrm{mg}$ vincristine (Hangzhou Minsheng Pharmaceutical Group Co., Ltd., Hangzhou, China) on day 1
Table I. Immunohistochemistry results of a lymph node biopsy specimen.

\begin{tabular}{lc} 
Immunohistochemical & Positive rate \\
\hline CD20 & ++ \\
TdT & - \\
MUM-1 & + \\
EBER & - \\
CD15 & - \\
CD30 & - \\
CD79a & ++ \\
CD10 & - \\
EMA & - \\
CD38 & \pm \\
Ki67 & $+/ 60 \%$ \\
UCHL1 & \pm \\
Pax-5 & + \\
ALK & + \\
CD3 & + \\
BCL-6 & + \\
kappa & lambda
\end{tabular}

TdT, terminal deoxynucleotidyl transferase; MUM, multiple myeloma oncogene; EBER, EBV-encoded region; EMA, epithelial membrane antigen; UCHL1, ubiquitin carboxyl-terminal esterase L1; Pax-5, paired box-5; ALK, anaplastic lymphoma receptor tyrosine kinase; BCL-6, B-cell lymphoma 6 protein;,$+<25 \%$; ++, 25-50\%; ,$+++ 50-75 \%$; ++++, $>75 \%$; -, negative; \pm , result not obtained.

and $40 \mathrm{mg} / \mathrm{m}^{2}$ prednisone (Shanghai Xinyi Pharmaceutical Co., Ltd., Shanghai, China) on days 1-5. The induction therapy was given every 4 weeks for a total of 6 cycles. Eight-cycle $375-\mathrm{mg} / \mathrm{m}^{2}$ rituximab infusion was subsequently administered as maintenance therapy. The R-mini-CHOP therapy was well tolerated, and myocardial biopsy confirmed a grade $0-1$ allograft rejection at 2.5 years after the previous rituximab maintenance therapy (Fig. 2). The patient remained in clinical remission, as shown by follow-up positron emission tomography (Biograoh MCT; Siemens AG, Munich, Germany) and computed tomography 1 and 5 years after R-mini-CHOP therapy (Fig. 3).

\section{Discussion}

The first heart transplantation was performed in 1967 (9), and PTLD was first reported in a kidney recipient in 1979 (10). PTLD is the most common malignancy second to skin/lip cancer in patients who have undergone solid organ transplantation, with an estimated incidence rate of 159/100,000 persons per year (11). Skin and splenic lymph nodes (56\%) are most frequently involved in PTLD at the time of presentation (8). In addition, non-renal transplant recipients, in particular heart and lung recipients, have a higher risk of developing PTLD than renal transplant recipients (12). To the best of our knowledge, the present study reports the first case of late-onset 

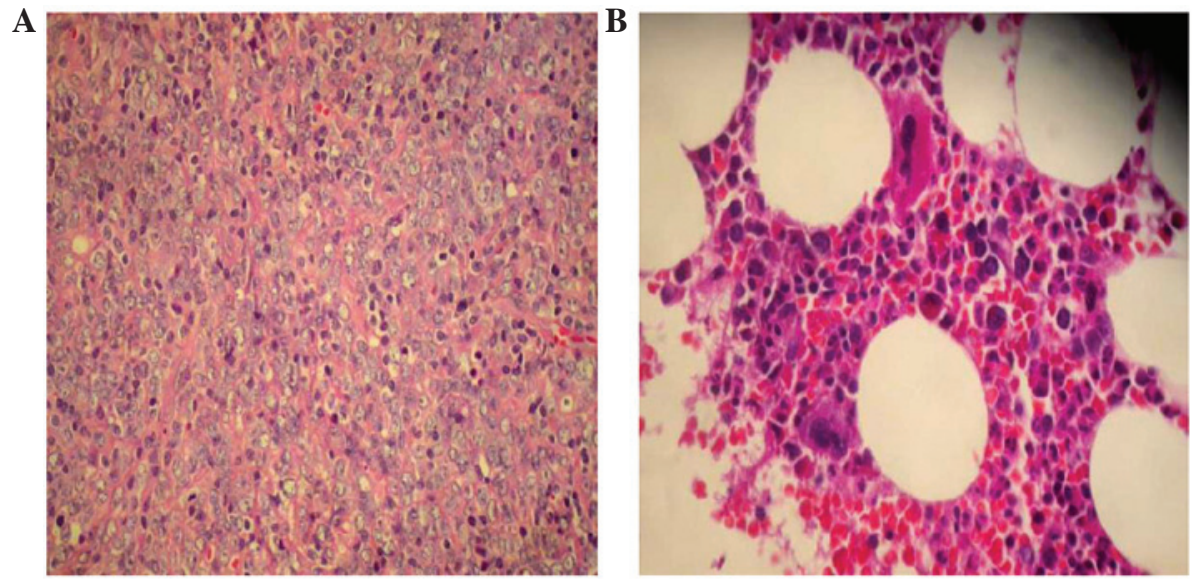

Figure 1. Clinical, radiologic and histologic presentations of post-transplant lymphoproliferative disorder. (A) Histology showing diffuse large B-cell lymphoma (magnification, $\mathrm{x} 400$ ); (B) Bone marrow examination showing mild marrow hyperplasia (magnification, $\mathrm{x} 400$ ).

PTLD following heart transplantation in a Chinese patient, although 200 cases of PTLD have been reported among heart recipients in the English literature.

PTLD is the overproliferation of lymphoid cells, with disease severity ranging from benign polyclonal histology to malignant lymphoma (13). The most common pathologies of PTLD include DLBCL and immunoblastic lymphoma (14), whereas T-cell lymphomas, Hodgkin disease and plasma cell neoplasms are less frequently encountered (15). Post-transplantation, patients are at the highest risk of developing early-onset PTLD, which occurs within the first postoperative year $(3,16,17)$. The early-onset subtype more frequently affects pediatric and younger adult patients as polymorphic lymphoid proliferation with a possible causative association with EBV infection (3), whereas the late-onset subtype, which manifests as monomorphic lymphomas of B-cells, or less often, T-cells, primarily occurs in older patients without a definitive association with EBV infection $(18,19)$. Late-onset PTLD patients (21\%) are more likely to be negative on the Epstein-Barr encoding region (EBER) in situ hybridization, compared with early-onset patients (10\%) (12).

As the EBV-associated B-cell tumor is understood to result from impaired immunity following immunosuppressive therapy, the reduction or cessation of immunosuppressive therapy is essential for the treatment of PTLD and may lead to spontaneous remission in early cases; however, reduced immunosuppression may elicit allograft rejection (4). Au et al (20) reported a case of brain PTLD (EBER+, CD20+, DLBCL) in a Chinese patient following heart transplantation, and the patient achieved complete remission following a reduction of immunosuppression. However, the patient succumbed to allograft rejection after 5 months. Intensive chemotherapy is typically required if reduced immunosuppression fails to control early-onset disease, and is the initial therapy for the majority of cases of late-onset PTLD (2).

An antracyclin-based multi-drug regimen, such as the CHOP regimen with granulocyte-colony stimulating factor (21), is used in the majority of transplantation centers. An underlying adverse effect of cytotoxic chemotherapy is enhanced hematotoxicity in patients with PTLD, as previous long-term immunosuppression may result in kidney and

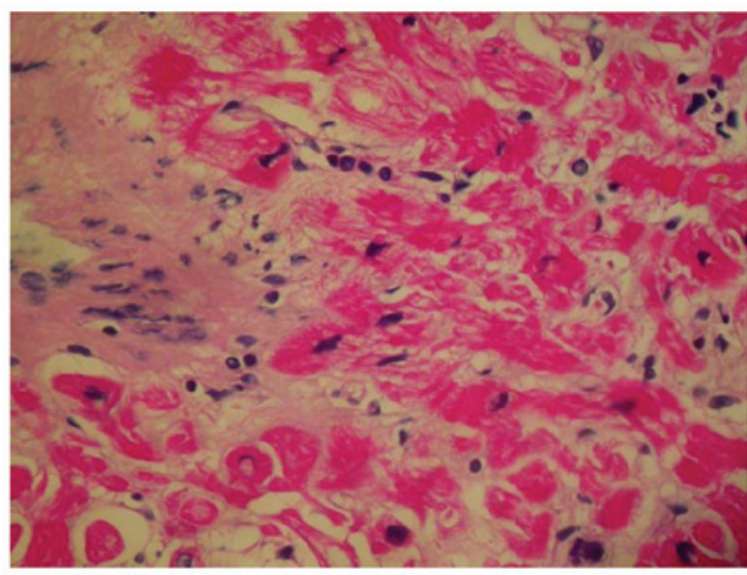

Figure 2. Histology of a myocardial biopsy specimen (magnification, $\mathrm{x} 400$ ) demonstrates clearly distributed myocardial longitudinal lines and horizontal stripes, infiltration of a number of lymphocytes into the interstitial microvessels, and absence of obvious myocardial necrosis.

marrow toxicities (22). These patients also have a higher susceptibility to infectious complications and sepsis, due to the underlying impairment of immunity (22). A low-dose CHOP, namely the mini-CHOP regimen, was developed by Windebank et al (23) and Gross et al (24) and is associated with reduced drug toxicity and better tolerability.

PTLD following solid organ transplantation is understood to be immunoreactive to CD20 in $>90 \%$ of cases, and DLBCL affects $\leq 75 \%$ of adult PTLD cases with monomorphic histology (25). The chimeric antibody rituximab, which specifically targets the CD20 antigen, has been incorporated in the treatment of PTLD in a number of case reports and retrospective studies (26-28). The use of rituximab may allow a reduction in chemotherapy dose and result in reduced cytoxicity, particularly in pediatric patients. In 5 out of 6 pediatric PTLD patients, rituximab combined with dose-reduced chemotherapy resulted in a complete response within an 8- to 29-month follow-up period, with limited toxicity (29). Gupta et al (13) reported that direct rituximab and reduced dose chemotherapy following the failure of reduced immunosuppression appeared to be effective as an alternative treatment of option for B-cell PTLD. Kusuki et al (30) reported that the 


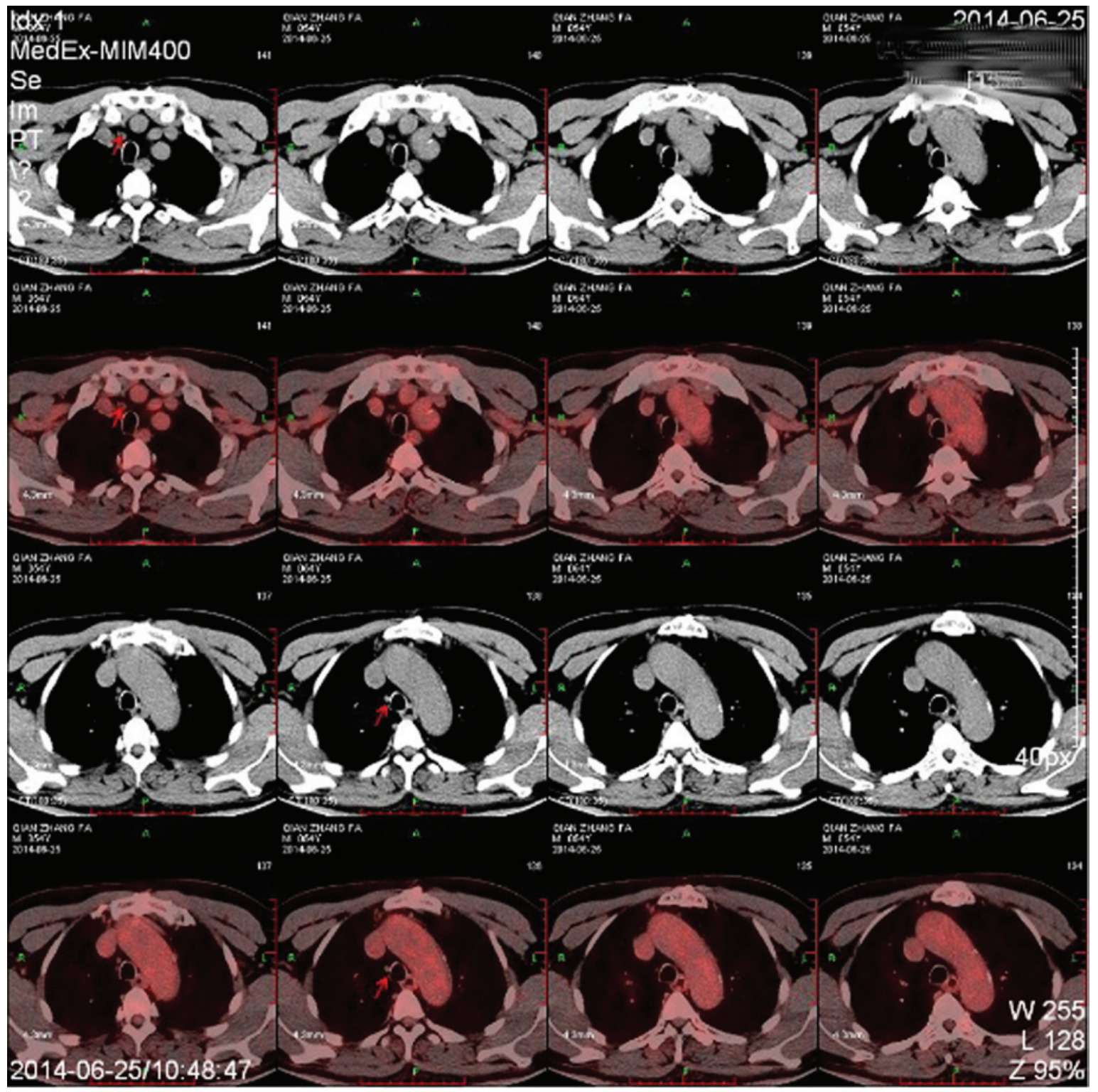

Figure 3. Positron emission tomography-computed tomography at the 5-year follow-up. The scans show no relapse of post-transplant lymphoproliferative disorder.

rituximab and combination chemotherapy regimen was effective in inducing and maintaining remission of PTLD. The present study is the first case report to evaluate the efficacy and safety of the R-mini-CHOP regimen in an adult PTLD patient following solid organ transplantation.

PTLD patients, in particular those suffering from the late-onset subtype, typically have an unfavorable prognosis, and approximately one-third of post-heart transplantation patients succumb to PTLD $(31,32)$. The survival probability of pediatric early-onset PTLD heart recipients varies among previous reports $(31,32)$, although remains low in adult late-onset patients. In a case study of 56 pediatric heart recipients with PTLD, the probability of survival was $75 \%$ at 1 year, 68\% at 3 years and $67 \%$ at 5 years after diagnosis (32). The patient in the current study exhibited clinical remission free of cytoxicity and allograft rejection at 5 years following R-mini-CHOP therapy for PTLD.

In conclusion, the patient in the present study presented EBV-negative, late-onset PTLD following heart transplantation. Reduced immunosuppression and the R-mini-CHOP regimen resulted in long-term clinical remission in the patient. This treatment regimen exhibited good tolerability and a good safety profile with respect to cytoxicity and allograft rejection. These results support the clinical practice of the management of B-cell PTLD in adult late-onset patients.

\section{References}

1. Gao SZ, Chaparro SV, Perlroth M, Montoya JG, Miller JL, DiMiceli S, Hastie T, Oyer PE and Schroeder J: Post-transplantation lymphoproliferative disease in heart and heart-lung transplant recipients: 30 -year experience at Stanford University. J Heart Lung Transplant 22: 505-514, 2003.

2. Taylor AL, Marcus R and Bradley JA: Post-transplant lymphoproliferative disorders (PTLD) after solid organ transplantation. Crit Rev Oncol Hematol 56: 155-167, 2005.

3. Wasson S, Zafar MN, Best J and Reddy HK: Post-transplantation lymphoproliferative disorder in heart and kidney transplant patients: A single-center experience. J Cardiovasc Pharmacol Ther 11: 77-83, 2006. 
4. Khedmat H, Alavian S and Taheri S: Significance of Epstein-Barr virus infection in the outcome of renal transplant patients with lymphoproliferative disorders. Ann Transplant 15: 40-44, 2010.

5. Poirel HA, Bernheim A, Schneider A, Meddeb M, Choquet S, Leblond V, Charlotte F, Davi F, Canioni D, Macintyre E, et al: Characteristic pattern of chromosomal imbalances in posttransplantation lymphoproliferative disorders: Correlation with histopathological subcategories and EBV status. Transplantation 80: 176-184, 2005.

6. Tsai DE, Hardy CL, Tomaszewski JE, Kotloff RM, Oltoff KM, Somer BG, Schuster SJ, Porter DL, Montone KT and Stadtmauer EA: Reduction in immunosuppression as initial therapy for posttransplant lymphoproliferative disorder: Analysis of prognostic variables and long-term follow-up of 42 adult patients. Transplantation 71: 1076-1088, 2001.

7. Herman J, Vandenberghe P, van den Heuvel I, Van Cleemput J, Winnepenninckx $\mathrm{V}$ and Van Damme-Lombaerts $\mathrm{R}$ : Successful treatment with rituximab of lymphoproliferative disorder in a child after cardiac transplantation. J Heart Lung Transplant 21: 1304-1309, 2002.

8. Campo E, Swerdlow SH, Harris NL, Pileri S, Stein H and Jaffe ES: The 2008 WHO classification of lymphoid neoplasms and beyond: Evolving concepts and practical applications. Blood 117: 5019-5032, 2011.

9. Barnard CN: The first transplantation of a human heart. Presse Med 75: 2815-2816, 1967 (In French).

10. Marker SC, Ascher NL, Kalis JM, Simmons RL, Najarian JS and Balfour HH Jr: Epstein-Barr virus antibody responses and clinical illness in renal transplant recipients. Surgery 85: 433-440, 1979.

11. Kinch A, Baecklund E, Backlin C, Ekman T, Molin D, Tufveson G, Fernberg P, Sundström C, Pauksens K and Enblad G: A population-based study of 135 lymphomas after solid organ transplantation: The role of Epstein-Barr virus, hepatitis $\mathrm{C}$ and diffuse large B-cell lymphoma subtype in clinical presentation and survival. Acta Oncol 53: 669-679, 2014.

12. Yousem SA, Randhawa P, Locker J, Paradis IL, Dauber JA, Griffith BP and Nalesnik MA: Posttransplant lymphoproliferative disorders in heart-lung transplant recipients: Primary presentation in the allograft. Hum Pathol 20: 361-369, 1989.

13. Gupta S, Fricker FJ, González-Peralta RP, Slayton WB, Schuler PM and Dharnidharka VR: Post-transplant lymphoproliferative disorder in children: Recent outcomes and response to dual rituximab/low-dose chemotherapy combination. Pediatr Transplant 14: 896-902, 2010.

14. Weissmann DJ, Ferry JA, Harris NL, Louis DN, Delmonico F and Spiro I: Posttransplantation lymphoproliferative disorders in solid organ recipients are predominantly aggressive tumors of host origin. Am J Clin Pathol 103: 748-755, 1995.

15. Schubert S, Abdul-Khaliq H, Lehmkuhl HB, Yegitbasi M, Reinke P, Kebelmann-Betzig C, Hauptmann K, Gross-Wieltsch U, Hetzer R and Berger F: Diagnosis and treatment of post-transplantation lymphoproliferative disorder in pediatric heart transplant patients. Pediatr Transplant 13: 54-62, 2009.

16. Shan D, Ledbetter JA and Press OW: Apoptosis of malignant human B cells by ligation of CD20 with monoclonal antibodies. Blood 91: 1644-1652, 1998.

17. Trofe J, Buell JF, Beebe TM, Hanaway MJ, First MR, Alloway RR, Gross TG, Succop P and Woodle ES: Analysis of factors that influence survival with post-transplant lymphoproliferative disorder in renal transplant recipients: The Israel Penn International Transplant Tumor Registry experience. Am J Transplant 5: 775-780, 2005

18. Khedmat $\mathrm{H}$ and Taheri S: Early versus late outset of lymphoproliferative disorders post-heart and lung transplantation: The PTLD. Int Survey. Hematol Oncol Stem Cell Ther 4: 10-16, 2011.
19. Webber SA, Naftel DC, Fricker FJ, Olesnevich P, Blume ED, Addonizio L, Kirklin JK and Canter CE; Pediatric Heart Transplant Study: Lymphoproliferative disorders after paediatric heart transplantation: A multi-institutional study. Lancet 367: 233-239, 2006.

20. Au WY, Lie AK, Kwong YL, Shek TW, Hawkins BR, Lai KN, Tang SC, Lo CM, Fan ST, Liu CL, et al: Post-transplantation lymphoproliferative disease in Chinese: The Queen Mary Hospital experience in Hong Kong. Leuk Lymphoma 43: $1403-1407,2002$

21. Garrett TJ, Chadburn A, Barr ML, Drusin RE, Chen JM, Schulman LL, Smith CR, Reison DS, Rose EA and Michler RE: Posttransplantation lymphoproliferative disorders treated with cyclophosphamide-doxorubicin-vincristine-prednisone chemotherapy. Cancer 72: 2782-2785, 1993.

22. Choquet S, Trappe R, Leblond V, Jäger U, Davi F and Oertel S: CHOP-21 for the treatment of post-transplant lymphoproliferative disorders (PTLD) following solid organ transplantation. Haematologica 92: 273-274, 2007.

23. Windebank K, Walwyn T, Kirk R, Parry G, Hasan A, Bown N and Wilkins B: Post cardiac transplantation lymphoproliferative disorder presenting as $\mathrm{t}(8 ; 14)$ Burkitt leukaemia/lymphoma treated with low intensity chemotherapy and rituximab. Pediatr Blood Cancer 53: 392-396, 2009.

24. Gross TG, Hinrichs SH, Winner J, Greiner TC, Kaufman SS, Sammut PH and Langnas AN: Treatment of post-transplant lymphoproliferative disease (PTLD) following solid organ transplantation with low-dose chemotherapy. Ann Oncol 9: 339-340, 1998.

25. Harris N, Swerdlow SH, Frizzera G and Knowles DM: Post-transplant lymphoproliferative disorders. IARC Press, Cheenra, 2001

26. Milpied N, Vasseur B, Parquet N, Garnier JL, Antoine C, Quartier P, Carret AS, Bouscary D, Faye A, Bourbigot B, et al: Humanized anti-CD20 monoclonal antibody (Rituximab) in post transplant B-lymphoproliferative disorder: A retrospective analysis on 32 patients. Ann Oncol 11 (Suppl 1): 113-116, 2000.

27. Oertel SH, Anagnostopoulos I, Bechstein WO, Liehr H and Riess HB: Treatment of posttransplant lymphoproliferative disorder with the anti-CD20 monoclonal antibody rituximab alone in an adult after liver transplantation: A new drug in therapy of patients with posttransplant lymphoproliferative disorder after solid organ transplantation?. Transplantation 69: 430-432, 2000.

28. Verschuuren EA, Stevens SJ, van Imhoff GW, Middeldorp JM, de Boer C, Koëter G, The TH and van Der Bij W: Treatment of posttransplant lymphoproliferative disease with rituximab: The remission, the relapse and the complication. Transplantation 73: 100-104, 2002

29. Orjuela M, Gross TG, Cheung YK, Alobeid B, Morris E and Cairo MS: A pilot study of chemoimmunotherapy (cyclophosphamide, prednisone and rituximab) in patients with post-transplant lymphoproliferative disorder following solid organ transplantation. Clin Cancer Res 9: 3945S-3952S, 2003.

30. Kusuki S, Hashii Y, Fukushima N, Takizawa S, Tokimasa S, Kogaki S, Ohta H, Tsuda E, Nakagawa A and Ozono K: Pediatric post-transplant diffuse large B cell lymphoma after cardiac transplantation. Int J Hematol 89: 209-213, 2009.

31. Boyle GJ, Michaels MG, Webber SA, Knisely AS, Kurland G, Cipriani LA, Griffith BP and Fricker FJ: Posttransplantation lymphoproliferative disorders in pediatric thoracic organ recipients. J Pediatr 131: 309-313, 1997.

32. Cohen AH, Sweet SC, Mendeloff E, Mallory GB Jr, Huddleston CB, Kraus M, Kelly M, Hayashi R and DeBaun MR: High incidence of posttransplant lymphoproliferative disease in pediatric patients with cystic fibrosis. Am J Respir Crit Care Med 161: 1252-1255, 2000 